\title{
SYNTHESIS AND SPECTRAL ANALYSIS OF AN ARRAY OF NOVEL 4-(4-MORPHOLINOPHENYL)-6-ARYL- PYRIMIDIN-2-AMINES
}

\author{
J.THANUSU, V.KANAGARAJAN, M. GOPALAKRISHNAN* \\ Synthetic Organic Chemistry Laboratory, Department of Chemistry, \\ Annamalai University, Annamalainagar-608 002, Tamil Nadu, India. \\ (Received: May 5, 2010 - Accepted: December 28, 2010)
}

\begin{abstract}
An array of newly synthesized novel4-(4-morpholinophenyl)-6-arylpyrimidin-2-amines (20-28) are synthesized from the respective (E)-1-4-morpholinophenyl)3-aryl-prop-2-en-1-ones (11-19) by the treatment of guanidine nitrate in refluxing ethanol catalyzed by lithium hydroxide and characterized by melting point, elemental analysis, MS, FT-IR, one-dimensional NMR $\left({ }^{1} \mathrm{H} \&{ }^{13} \mathrm{C}\right)$ spectroscopic data.
\end{abstract}

Keywords: 1-(4-morpholinophenyl) ethanone; (E)-1-4-morpholinophenyl)-3-aryl-prop-2-en-1-ones; 4-(4-morpholinophenyl)-6-arylpyrimidin-2-amines; Guanidine nitrate; Synthesis.

\section{INTRODUCTION}

Pyrimidines are the basic nucleus in nucleic acids and have been associated with a number of biological activities. Some notable biological activity of pyrimidine derivatives include adenosine receptor antagonists [1], kinase inhibitors [2], analgesic [3], anti-inflammatory [3], inhibitors of cyclin-Dependent kinases 1 and 2 [4], calcium channel antagonist [5], antihistaminic [6], antitubercular [7] activities. Substituted aminopyrimidine nuclei are common in marketed drugs such as anti-atherosclerotic aronixil, anti-histaminic thonzylamine, anti-anxielytic buspirone, anti-psoriatic enazadrem, and other medicinally relevant compounds.

Morpholine is a simple heterocyclic compound with a great industrial importance. It is used as anticorrosive agent and as chemical intermediate: catalyst, solvent, antioxidant, in the production of various pharmaceuticals and pesticides. 4-Phenyl morpholine derivatives [8] are reported to possess antimicrobial, anti-inflammatory and central nervous system activities. Linezolide (commercially available antimicrobial) also possess a 4-phenyl-morpholine substituent. They are reported to exert a number of important physiological activities such as antidiabetic [9], antiemetic [10], platelet aggregation inhibitors, antihyperlipoproteinemics [9], bronchodilators, growth stimulats [11] and antidepressants [12]. These were also used in the treatment of inflammatory diseases, pain, migraine and asthma [13].

Recently, we exploited the synthesis of 6-aryl-1,2,4,5-tetrazinane-3thiones [14], fused indazoles [15], 3,4-dihydropyrimidin-2(1H)-ones/thiones [16], pyrimidine and 2,6-diarylpiperidin-4-one derivatives [17-19] with a view to incorporate various other bioactive heterocyclic nucleus such as 1,2,3-selenadiazoles, 1,2,3-thiadiazoles, diazepans intact for evaluation of associated antibacterial and antifungal activities. In view of the above and as part of the ongoing research on antimicrobials [17], we planned to synthesize a system, which comprises both $\mathrm{N}$-functionalized morpholine and 2-amino4,6-diarylpyrimidine components together to give a compact structure like title 4-(4-morpholinophenyl)-6-arylpyrimidin-2-amines.

\section{EXPERIMENTAL}

Performing TLC assessed the reactions and the purity of the products. All the reported melting points were taken in open capillaries and were uncorrected. IR spectra were recorded in $\mathrm{KBr}$ (pellet forms) on a Nicolet-Avatar-330 FT-IR spectrophotometer and note worthy absorption values $\left(\mathrm{cm}^{-1}\right)$ alone are listed. ${ }^{1} \mathrm{H}$ and ${ }^{13} \mathrm{C}$ NMR spectra were recorded at $400 \mathrm{MHz}$ and $100 \mathrm{MHz}$ respectively on Bruker AMX 400 NMR spectrometer using DMSO- $d$ as solvent. The ESI +ve MS spectra were recorded on a Bruker Daltonics LC-MS spectrometer. Satisfactory microanalysis was obtained on Carlo Erba $1106 \mathrm{CHN}$ analyzer.

General procedure for the synthesis of (E)-1-4-morpholinophenyl)-3aryl-prop-2-en-1-ones (11-19)

To an ethanolic solution of 1-(4-morpholinophenyl) ethanone (0.001 mol) and substituted benzaldehyde $(0.001 \mathrm{~mol})$, aqueous sodium hydroxide $(0.005$ mol) was added drop wise with stirring on a mechanical stirrer for 10 minutes and stirring was continued for 2-6 h. After completion of reaction, the crude product isolated by suction was washed with water, dried and recrystallized from ethanol.

(E)-1-(4-morpholinophenyl)-3-phenyl-prop-2-en-1-one (11)

Reaction time: $4 \mathrm{~h} ; \mathrm{IR}(\mathrm{KBr}) v\left(\mathrm{~cm}^{-1}\right)$ : 3007, 2962, 2924, 2852, 1646, 1606 , 1190,$769 ;{ }^{1} \mathrm{H}$ NMR $(\delta \mathrm{ppm}): 3.33-3.36$ (t, $\left.4 \mathrm{H}, \mathrm{N}\left(\mathrm{CH}_{2}\right)_{2}, J=4.7 \mathrm{~Hz}\right), 3.87-3.89$ (t, $\left.4 \mathrm{H}, \mathrm{O}\left(\mathrm{CH}_{2}\right)_{2}, J=4.7 \mathrm{~Hz}\right), 6.93-6.95\left(\mathrm{~d}, 1 \mathrm{H}, \mathrm{H}_{2}, J=8.9 \mathrm{~Hz}\right) ; 7.38-7.82(\mathrm{~m}, 10 \mathrm{H}$, $\left.\mathrm{H}_{\text {arom }}\right) ; 8.01-8.03\left(\mathrm{~d}, 1 \mathrm{H}, \mathrm{H}_{3}, J=8.9 \mathrm{~Hz}\right) ;{ }^{13} \mathrm{C} \mathrm{NMR}(\delta \mathrm{ppm}): 47.7 \mathrm{~N}\left(\mathrm{CH}_{2}\right), 66.5$ $\mathrm{O}\left(\mathrm{CH}_{2}\right), 122.3 \mathrm{C}-2,143.2 \mathrm{C}-3,113.6,128.8-130.3-\mathrm{C}_{\text {arom }}, 128.2,135.4,154.1$ ipso-C, $188.1 \mathrm{C}-1$.

(E)-1-(4-morpholinophenyl)-3-p-tolyl-prop-2-en-1-one (12)

Reaction time: $5 \mathrm{~h}$; IR $(\mathrm{KBr}) \vee\left(\mathrm{cm}^{-1}\right): 3012,2923,2924,2851,1645,1600$, 1194,$810 ;{ }^{1} \mathrm{H}$ NMR $(\delta \mathrm{ppm}): 1.57\left(\mathrm{~s}, 3 \mathrm{H}, \mathrm{CH}_{3}\right.$ at phenyl ring), 3.32-3.35 (t, $4 \mathrm{H}$, $\left.\mathrm{N}\left(\mathrm{CH}_{2}\right)_{2}, J=4.8 \mathrm{~Hz}\right), 3.86-3.89\left(\mathrm{t}, 4 \mathrm{H}, \mathrm{O}\left(\mathrm{CH}_{2}\right)_{2}, J=4.8 \mathrm{~Hz}\right), 6.92-6.94\left(\mathrm{~d}, 1 \mathrm{H}, \mathrm{H}_{2}\right.$, $J=8.8 \mathrm{~Hz}) ; 7.21-7.80\left(\mathrm{~m}, 9 \mathrm{H}, \mathrm{H}_{\text {arom }}\right) ; 8.00-8.02\left(\mathrm{~d}, 1 \mathrm{H}, \mathrm{H}_{3}, J=8.8 \mathrm{~Hz}\right) ;{ }^{13} \mathrm{C}$ NMR $(\delta \mathrm{ppm}): 21.0 \mathrm{CH}_{3}$ at phenyl ring, $47.7 \mathrm{~N}\left(\mathrm{CH}_{2}\right), 66.5 \mathrm{O}\left(\mathrm{CH}_{2}\right), 121.2 \mathrm{C}-2,143.3$ C-3, 113.5, 129.3-130.5 - $\mathrm{C}_{\text {arom }}, 128.2,132.7,140.5,154.1$ ipso-C, $188.2 \mathrm{C}-1$.

(E)- 3-(4-chlorophenyl)-1-(4-morpholinophenyl)prop-2-en-1-one (13)

Reaction time: $5 \mathrm{~h}$; IR $(\mathrm{KBr}) \vee\left(\mathrm{cm}^{-1}\right): 3087,2967,2920,2859,1597$, $1654,1202,817 ;{ }^{1} \mathrm{H}$ NMR $(\delta \mathrm{ppm}): 3.34-3.37\left(\mathrm{t}, 4 \mathrm{H}, \mathrm{N}\left(\mathrm{CH}_{2}\right)_{2}, J=4.7 \mathrm{~Hz}\right)$, 3.89-3.91 (t, $\left.4 \mathrm{H}, \mathrm{O}\left(\mathrm{CH}_{2}\right)_{2}, J=4.8 \mathrm{~Hz}\right), 6.97-6.99$ (d, $\left.1 \mathrm{H}, \mathrm{H}_{2}, J=8.8 \mathrm{~Hz}\right) ; 7.35-$ $7.76\left(\mathrm{~m}, 9 \mathrm{H}, \mathrm{H}_{\text {arom }}\right) ; 8.00-8.02\left(\mathrm{~d}, 1 \mathrm{H}, \mathrm{H}_{3}, J=8.9 \mathrm{~Hz}\right) ;{ }^{13} \mathrm{C} \mathrm{NMR}(\delta \mathrm{ppm}): 47.6$ $\mathrm{N}\left(\mathrm{CH}_{2}\right), 66.5 \mathrm{O}\left(\mathrm{CH}_{2}\right), 121.2 \mathrm{C}-2,141.8 \mathrm{C}-3,113.5,129.4-130.6-\mathrm{C}_{\text {arom }}$, 129.1,133.8,135.0,154.0 ipso-C, $192.2 \mathrm{C}-1$. (14)

(E)- 3-(4-methoxyphenyl)-1-(4-morpholinophenyl)prop-2-en-1-one

Reaction time: $4 \mathrm{~h}$; IR $(\mathrm{KBr}) \vee\left(\mathrm{cm}^{-1}\right): 3010,2961,2918,2841,1645$, 1601,$1225 ;{ }^{1} \mathrm{H}$ NMR $(\delta \mathrm{ppm}): 3.32-3.35\left(\mathrm{t}, 4 \mathrm{H}, \mathrm{N}\left(\mathrm{CH}_{2}\right)_{2}, J=4.8 \mathrm{~Hz}\right), 3.87-3.90$ $\left(\mathrm{t}, 4 \mathrm{H}, \mathrm{O}\left(\mathrm{CH}_{2}\right), J=4.8 \mathrm{~Hz}\right), 3.86\left(s, 3 \mathrm{H}, \mathrm{OCH}_{3}\right.$ at phenyl ring), 7.59-7.61 (d, $\left.1 \mathrm{H}, \mathrm{H}_{2}, J=8.6 \mathrm{~Hz}\right) ; 6.92-7.46$ \& 7.75-7.79 (m, $\left.9 \mathrm{H}, \mathrm{H}_{\text {arom }}\right) ; 8.00-8.02\left(\mathrm{~d}, 1 \mathrm{H}, \mathrm{H}_{3}\right.$, $J=8.7 \mathrm{~Hz}) ;{ }^{13} \mathrm{C}$ NMR $(\delta \mathrm{ppm}): 47.6 \mathrm{~N}\left(\mathrm{CH}_{2}\right), 55.3 \mathrm{OCH}_{3}$ at phenyl ring, 66.5 $\mathrm{O}\left(\mathrm{CH}_{2}\right), 119.6 \mathrm{C}-2,143.1 \mathrm{C}-3,113.5,129.2-130.4-\mathrm{C}_{\text {arom }}{ }^{3}, 127.9,129.9,153.9$, 161.3 ipso-C, $187.8 \mathrm{C}-1$.

(E)- 3-(4-fluorophenyl)-1-(4-morpholinophenyl)prop-2-en-1-one (15)

Reaction time: $4 \mathrm{~h}$; IR $(\mathrm{KBr}) \vee\left(\mathrm{cm}^{-1}\right): 3009,2969,2919,2849,1650,1602$, $1227 ;{ }^{1} \mathrm{H}$ NMR $(\delta \mathrm{ppm}): 3.33-3.36\left(\mathrm{t}, 4 \mathrm{H}, \mathrm{N}\left(\mathrm{CH}_{2}\right)_{2}, J=4.7 \mathrm{~Hz}\right), 3.87-3.89(\mathrm{t}, 4 \mathrm{H}$, $\left.\mathrm{O}\left(\mathrm{CH}_{2}\right)_{2}, J=4.8 \mathrm{~Hz}\right), 6.93-6.95\left(\mathrm{~d}, 1 \mathrm{H}, \mathrm{H}_{2}, J=8.9 \mathrm{~Hz}\right) ; 7.08-7.78\left(\mathrm{~m}, 9 \mathrm{H}, \mathrm{H}_{\text {arom }}\right)$; $8.00-8.02\left(\mathrm{~d}, 1 \mathrm{H}, \mathrm{H}_{3}, J=8.9 \mathrm{~Hz}\right) ;{ }^{13} \mathrm{C}$ NMR $(\delta \mathrm{ppm}): 47.5 \mathrm{~N}\left(\mathrm{CH}_{2}\right), 66.5 \mathrm{O}\left(\mathrm{CH}_{2}\right)$, 121.6 C-2, 141.9 C-3, 113.4, 115.8, 130.1,131.5- $\mathrm{C}_{\text {arom }}, 128.8,130.6,154.1$, 162.5 ipso-C, 187.8 C-1.

(E)- 3-(4-bromophenyl)-1-(4-morpholinophenyl)prop-2-en-1-one (16)

Reaction time: $2 \mathrm{~h}$; IR $(\mathrm{KBr}) v\left(\mathrm{~cm}^{-1}\right): 3001,2960,2923,2845,1657,1612$, $1227 ;{ }^{1} \mathrm{H}$ NMR $(\delta \mathrm{ppm}): 3.32-3.35\left(\mathrm{t}, 4 \mathrm{H}, \mathrm{N}\left(\mathrm{CH}_{2}\right), J=4.5 \mathrm{~Hz}\right), 3.86-3.87(\mathrm{t}, 4 \mathrm{H}$, $\left.\mathrm{O}\left(\mathrm{CH}_{2}\right)_{2}, J=4.6 \mathrm{~Hz}\right), 6.94-6.96\left(\mathrm{~d}, 1 \mathrm{H}, \mathrm{H}_{2}, J=8.8 \mathrm{~Hz}\right) ; 7.18-7.82\left(\mathrm{~m}, 9 \mathrm{H}, \mathrm{H}_{\text {arom }}\right)$; $8.01-8.03\left(\mathrm{~d}, 1 \mathrm{H}, \mathrm{H}_{3}, J=8.6 \mathrm{~Hz}\right) ;{ }^{13} \mathrm{C}$ NMR $(\delta \mathrm{ppm}): 47.9 \mathrm{~N}\left(\mathrm{CH}_{2}\right), 65.6 \mathrm{O}\left(\mathrm{CH}_{2}\right)$, $121.8 \mathrm{C}-2,142.3 \mathrm{C}-3,113.8,115.1,130.7,131.7-\mathrm{C}_{\text {arom }}, 128.5,131.2,154.7$, 162.7 ipso-C, 188.8 C-1. 
(E)-1-(4-morpholinophenyl)-3-(3-nitrophenyl)prop-2-en-1-one (17)

Reaction time: $6 \mathrm{~h}$; IR $(\mathrm{KBr}) \vee\left(\mathrm{cm}^{-1}\right)$ : 3087, 2966, 2923, 2862, 1651, 1608 , 1224; ${ }^{1} \mathrm{H}$ NMR $(\delta \mathrm{ppm}): 3.36-3.38\left(\mathrm{t}, 4 \mathrm{H}, \mathrm{N}\left(\mathrm{CH}_{2}\right)_{2}, J=4.5 \mathrm{~Hz}\right), 3.88-3.90(\mathrm{t}, 4 \mathrm{H}$, $\left.\mathrm{O}\left(\mathrm{CH}_{2}\right)_{2}, J=4.6 \mathrm{~Hz}\right), 6.95-6.97\left(\mathrm{~d}, 1 \mathrm{H}, \mathrm{H}_{2}, J=8.9 \mathrm{~Hz}\right) ; 7.27-7.91 \& 8.23-8.25(\mathrm{~m}$, $\left.9 \mathrm{H}, \mathrm{H}_{\text {arom }}\right) ; 8.03-8.05\left(\mathrm{~d}, 1 \mathrm{H}, \mathrm{H}_{3}, J=8.9 \mathrm{~Hz}\right) ;{ }^{13} \mathrm{C}$ NMR $(\delta \mathrm{ppm}): 47.3 \mathrm{~N}\left(\mathrm{CH}_{2}\right)$ $66.9 \mathrm{O}\left(\mathrm{CH}_{2}\right), 122.0 \mathrm{C}-2,140.1 \mathrm{C}-3,113.3,124.2-134.2-\mathrm{C}_{\text {an }}, 128.9,137.1$, 148.7, 154.3 ipso-C, 187.8 C-1.

(E)-1-(4-morpholinophenyl)-3-(3-chlorophenyl)prop-2-en-1-one (18)

Reaction time: $6 \mathrm{~h}$; IR $(\mathrm{KBr}) \vee\left(\mathrm{cm}^{-1}\right): 3093,2969,2928,2857,1593$, $1652,1212,830 ;{ }^{1} \mathrm{H}$ NMR $(\delta \mathrm{ppm}): 3.33-3.36\left(\mathrm{t}, 4 \mathrm{H}, \mathrm{N}\left(\mathrm{CH}_{2}\right)_{2}, J=4.6 \mathrm{~Hz}\right)$, 3.89-3.91 (t, $\left.4 \mathrm{H}, \mathrm{O}\left(\mathrm{CH}_{2}\right)_{2}, J=4.6 \mathrm{~Hz}\right), 6.96-6.98\left(\mathrm{~d}, 1 \mathrm{H}, \mathrm{H}_{2}, J=8.9 \mathrm{~Hz}\right) ; 7.33-$ $7.81\left(\mathrm{~m}, 9 \mathrm{H}, \mathrm{H}_{\text {arom }}\right) ; 7.98-8.00\left(\mathrm{~d}, 1 \mathrm{H}, \mathrm{H}_{3}, J=8.7 \mathrm{~Hz}\right) ;{ }^{13} \mathrm{C}$ NMR $(\delta \mathrm{ppm}): 47.8$ $\mathrm{N}\left(\mathrm{CH}_{2}\right), 66.4 \mathrm{O}\left(\mathrm{CH}_{2}\right), 121.2 \mathrm{C}-2,141.6 \mathrm{C}-3,113.3,128.8-130.1-\mathrm{C}_{\text {arom }}$, 129.3,133.7,145.2,154.1 ipso-C, 192.3 C-1.

(E)-1-(4-morpholinophenyl)-3-(3-fluorophenyl)prop-2-en-1-one (19)

Reaction time: $5 \mathrm{~h}$; IR $(\mathrm{KBr}) \vee\left(\mathrm{cm}^{-1}\right)$ : 3018, 2974, 2924, 2843, 1649, 1605, 1226; ${ }^{1} \mathrm{H}$ NMR $(\delta \mathrm{ppm}): 3.33-3.36\left(\mathrm{t}, 4 \mathrm{H}, \mathrm{N}\left(\mathrm{CH}_{2}\right), J=4.8 \mathrm{~Hz}\right), 3.86-3.88$ (t, $4 \mathrm{H}$, $\left.\mathrm{O}\left(\mathrm{CH}_{2}\right)_{2}, J=4.7 \mathrm{~Hz}\right), 6.92-6.94\left(\mathrm{~d}, 1 \mathrm{H}, \mathrm{H}_{2}, J=8.8 \mathrm{~Hz}\right) ; 7.18-7.68\left(\mathrm{~m}, 9 \mathrm{H}, \mathrm{H}_{\text {arom }}\right) ;$ 7.92-7.94 (d, $\left.1 \mathrm{H}, \mathrm{H}_{3}, J=8.7 \mathrm{~Hz}\right) ;{ }^{13} \mathrm{C}$ NMR $(\delta \mathrm{ppm}): 47.5 \mathrm{~N}\left(\mathrm{CH}_{2}\right), 66.6 \mathrm{O}\left(\mathrm{CH}_{2}\right)$, $121.3 \mathrm{C}-2,141.8 \mathrm{C}-3,113.4,125.3-130.1-\mathrm{C}_{\text {arom }}, 128.6,130.5,154.3,162.7$ ipso-C, $187.5 \mathrm{C}-1$

General method for the synthesis of 4-(4-morpholinophenyl)-6-arylpyrimidin-2-amines (20-28)

A mixture of (E)-1-(4-morpholinophenyl)-3-aryl-prop-2-en-1-ones (1119) $(0.001 \mathrm{~mol})$ and guanidine nitrate $(0.001 \mathrm{~mol})$ in ethanol $(50 \mathrm{ml})$ was refluxed, while a solution of lithium hydroxide $(0.005 \mathrm{~mol})$ in water $(10 \mathrm{ml})$ was added portion wise for $1 \mathrm{~h}$. Re-fluxing was continued for further $4 \mathrm{~h}$ and the mixture was poured into ice cold water. The formed solid was separated by filtration, and purified by column chromatography using silica gel (100200 mesh), with ethyl acetate -Petroleum ether (bp40-60) in the ratio $(2: 8)$ as eluent.

4-(4-morpholinophenyl)-6-phenylpyrimidin-2-amine (20) IR ( $\mathrm{KBr})$ $\left(\mathrm{cm}^{-1}\right): 3355,3459,3060,2961,2920,1661,1599,1229,928,824,776,697$, 634; ${ }^{1} \mathrm{H}$ NMR $(\delta \mathrm{ppm}): 3.33-3.38\left(\mathrm{t}, 4 \mathrm{H}, \mathrm{N}\left(\mathrm{CH}_{2}\right)_{2}, J=4.7 \mathrm{~Hz}\right), 3.88-3.89$ (t, $4 \mathrm{H}$, $\left.\mathrm{O}\left(\mathrm{CH}_{2}\right)_{2}, J=4.8 \mathrm{~Hz}\right), 5.23\left(\mathrm{~s}, 2 \mathrm{H}, \mathrm{NH}_{2}\right), 7.38-7.85(\mathrm{~m}, 10 \mathrm{H}, \mathrm{H})$, the signal for $\mathrm{H}-5$ proton may be merged with the aromatic protons; ${ }^{13} \mathrm{C}$ NMR $(\delta \mathrm{ppm})$ : $46.3 \mathrm{~N}\left(\mathrm{CH}_{2}\right), 67.3 \mathrm{O}\left(\mathrm{CH}_{2}\right), 103.4 \mathrm{C}-5,163.8 \mathrm{C}-2,164.7 \mathrm{C}-6,165.0 \mathrm{C}-4,127.0-$ $131.5-\mathrm{C}_{\text {arom }}, 142.1,153.9$, ipso-C.

4-(4-morpholinophenyl)-6-p-tolylpyrimidin-2-amine (21) IR (KBr) $\left(\mathrm{cm}^{-1}\right): 3432,3200,2967,2923,1625,1599,1229,928,815,645 ;{ }^{1} \mathrm{H}$ NMR $(\delta$ ppm): 2.31 (s, $\left.3 \mathrm{H}, \mathrm{CH}_{3}\right), 3.34-3.38\left(\mathrm{t}, 4 \mathrm{H}, \mathrm{N}\left(\mathrm{CH}_{2}\right)_{2}, J=4.8 \mathrm{~Hz}\right), 3.86-3.89$ (t, $4 \mathrm{H}$, $\left.\mathrm{O}\left(\mathrm{CH}_{2}\right), J=4.8 \mathrm{~Hz}\right), 5.25\left(\mathrm{~s}, 2 \mathrm{H}, \mathrm{NH}_{2}\right), 7.20-8.12(\mathrm{~m}, 9 \mathrm{H}, \mathrm{H}$ ), the signal for $\mathrm{H}-5$ proton may be merged with the aromatic protons; ${ }^{13} \mathrm{C}$ NMR $(\delta \mathrm{ppm}): 25.4$ $\mathrm{CH}_{3}, 46.7 \mathrm{~N}\left(\mathrm{CH}_{2}\right), 67.5 \mathrm{O}\left(\mathrm{CH}_{2}\right), 104.1 \mathrm{C}-5,163.8 \mathrm{C}-2,164.1 \mathrm{C}-6,164.3 \mathrm{C}-4$, 126.0-131.4-C $\mathrm{C}_{\text {arom }}, 143.8,152.4$, ipso-C.

4-(4-chlorophenyl)-6-(4-morpholinophenyl)pyrimidin-2-amine (22) IR (KBr) $\left(\mathrm{cm}^{-1}\right): 3396,3217,3027,2962,2920,1656,1229,930,819,684 ;{ }^{1} \mathrm{H}$ NMR $(\delta \mathrm{ppm}): 3.35-3.39\left(\mathrm{t}, 4 \mathrm{H}, \mathrm{N}\left(\mathrm{CH}_{2}\right), J=4.7 \mathrm{~Hz}\right), 3.89-3.91\left(\mathrm{t}, 4 \mathrm{H}, \mathrm{O}\left(\mathrm{CH}_{2}\right)\right.$, $J=4.7 \mathrm{~Hz}), 5.28\left(\mathrm{~s}, 2 \mathrm{H}, \mathrm{NH}_{2}\right), 7.26-7.85(\mathrm{~m}, 9 \mathrm{H}, \mathrm{H}$ ), the signal for $\mathrm{H}-5$ proton may be merged with the aromatic protons; ${ }^{13} \mathrm{C}$ NMR $(\delta \mathrm{ppm}): 47.6 \mathrm{~N}\left(\mathrm{CH}_{2}\right)$, $66.5 \mathrm{O}\left(\mathrm{CH}_{2}\right), 104.5 \mathrm{C}-5,163.8 \mathrm{C}-2,164.1 \mathrm{C}-6,164.7 \mathrm{C}-4,127.0-139.1-\mathrm{C}_{\text {arom }}$, 141.5, 152.5, ipso-C.

4-(4-methoxyphenyl)-6-(4-morpholinophenyl)pyrimidin-2-amine (23) IR (KBr) $\left(\mathrm{cm}^{-1}\right): 3447,3200,2972,2922,1659,1600,1243,929,821,607 ;{ }^{1} \mathrm{H}$ NMR $(\delta \mathrm{ppm}): 3.32-3.35\left(\mathrm{t}, 4 \mathrm{H}, \mathrm{N}\left(\mathrm{CH}_{2}\right), J=4.6 \mathrm{~Hz}\right), 3.86\left(\mathrm{~s}, 3 \mathrm{H}, \mathrm{OCH}_{3}\right), 3.87-$ $3.90\left(\mathrm{t}, 4 \mathrm{H}, \mathrm{O}\left(\mathrm{CH}_{2}\right), J=4.8 \mathrm{~Hz}\right), 5.26\left(\mathrm{~s}, 2 \mathrm{H}, \mathrm{NH}_{2}\right), 7.18-7.82(\mathrm{~m}, 9 \mathrm{H}, \mathrm{H})$, the signal for $\mathrm{H}-5$ proton may be merged with the aromatic protons; ${ }^{13} \mathrm{C}$ NMR $(\delta$ ppm): $46.7 \mathrm{~N}\left(\mathrm{CH}_{2}\right), 55.0 \mathrm{OCH}_{3}, 67.3 \mathrm{O}\left(\mathrm{CH}_{2}\right), 103.9 \mathrm{C}-5,163.7 \mathrm{C}-2,164.7 \mathrm{C}-6$, 165.0 C-4, 127.5-142.1 - $\mathrm{C}_{\text {arom }}, 153.8,154.2$ ipso-C

4-(4-fluorophenyl)-6-(4-morpholinophenyl)pyrimidin-2-amine (24) IR (KBr) $\left(\mathrm{cm}^{-1}\right): 3434,3200,2967,2923,1624,1599,1226,928,815,645 ;{ }^{1} \mathrm{H}$ NMR ( $\delta$ ppm): $3.34-3.36\left(\mathrm{t}, 4 \mathrm{H}, \mathrm{N}\left(\mathrm{CH}_{2}\right), J=4.7 \mathrm{~Hz}\right), 3.87-3.88\left(\mathrm{t}, 4 \mathrm{H}, \mathrm{O}\left(\mathrm{CH}_{2}\right)\right.$ $J=4.8 \mathrm{~Hz}), 5.29\left(\mathrm{~s}, 2 \mathrm{H}, \mathrm{NH}_{2}\right), 7.30-8.03\left(\mathrm{~m}, 9 \mathrm{H}, \mathrm{H}_{\text {arom }}\right)$, the signal for $\mathrm{H}-5$ proton may be merged with the aromatic protons; ${ }^{13} \mathrm{C}$ NMR $(\delta \mathrm{ppm}): 46.6 \mathrm{~N}\left(\mathrm{CH}_{2}\right)$, $67.3 \mathrm{O}\left(\mathrm{CH}_{2}\right), 103.5 \mathrm{C}-5,163.8 \mathrm{C}-2,164.2 \mathrm{C}-6,164.4 \mathrm{C}-4,126.5-140.0-\mathrm{C}_{\text {arom }}$, 140.6, 154.0 ipso-C

4-(4-bromophenyl)-6-(4-morpholinophenyl)pyrimidin-2-amine (25) IR $(\mathrm{KBr})\left(\mathrm{cm}^{-1}\right): 3398,3219,3029,2965,2922,1659,1231,934,821,687 ;{ }^{1} \mathrm{H}$ $\operatorname{NMR}\left(\delta\right.$ ppm): $3.36-3.39\left(\mathrm{t}, 4 \mathrm{H}, \mathrm{N}\left(\mathrm{CH}_{2}\right)_{2}, J=4.8 \mathrm{~Hz}\right), 3.87-3.91\left(\mathrm{t}, 4 \mathrm{H}, \mathrm{O}\left(\mathrm{CH}_{2}\right)_{2}\right.$, $J=4.8 \mathrm{~Hz}), 5.29\left(\mathrm{~s}, 2 \mathrm{H}, \mathrm{NH}_{2}\right), 7.29-7.87\left(\mathrm{~m}, 9 \mathrm{H}, \mathrm{H}_{\text {arom }}\right)$, the signal for $\mathrm{H}-5$ proton may be merged with the aromatic protons; ${ }^{13} \mathrm{C}$ NMR $(\delta \mathrm{ppm}): 47.8 \mathrm{~N}\left(\mathrm{CH}_{2}\right)$, $66.6 \mathrm{O}\left(\mathrm{CH}_{2}\right), 104.8 \mathrm{C}-5,163.9 \mathrm{C}-2,164.3 \mathrm{C}-6,164.8 \mathrm{C}-4,127.5-139.7-\mathrm{C}_{\text {arom }}$, 152.6, 141.6 ipso-C. 4-(4-morpholinophenyl)-6-(3-nitrophenyl)pyrimidin-2-amine (26) IR $(\mathrm{KBr})\left(\mathrm{cm}^{-1}\right): 3400,3200,3060,2961,2920,1661,1566,1229,928,824,776$, 697; ${ }^{1} \mathrm{H}$ NMR $(\delta \mathrm{ppm}): 3.36-3.39\left(\mathrm{t}, 4 \mathrm{H}, \mathrm{N}\left(\mathrm{CH}_{2}\right)_{2}, J=4.6 \mathrm{~Hz}\right), 3.88-3.92(\mathrm{t}, 4 \mathrm{H}$, $\left.\mathrm{O}\left(\mathrm{CH}_{2}\right), J=4.6 \mathrm{~Hz}\right), 5.28\left(\mathrm{~s}, 2 \mathrm{H}, \mathrm{NH}_{2}\right), 7.27-8.28(\mathrm{~m}, 9 \mathrm{H}, \mathrm{H}$ ), the signal for $\mathrm{H}-5$ proton may be merged with the aromatic protons; ${ }^{13} \mathrm{C}$ NMR $(\delta \mathrm{ppm}): 47.3$ $\mathrm{N}\left(\mathrm{CH}_{2}\right), 67.6 \mathrm{O}\left(\mathrm{CH}_{2}\right), 104.4 \mathrm{C}-5,163.8 \mathrm{C}-2,164.2 \mathrm{C}-6,164.4 \mathrm{C}-4,125.2-$ $131.5-\mathrm{C}_{\text {rom }}, 146.7,153.9$ ipso-C.

4-(3-chlorophenyl)-6-(4-morpholinophenyl)pyrimidin-2-amine (27) IR $(\mathrm{KBr})\left(\mathrm{cm}^{-1}\right): 3398,3219,3028,2963,2923,1655,1228,929,817,686 ;{ }^{1} \mathrm{H}$ NMR ( $\delta$ ppm): $3.36-3.38\left(\mathrm{t}, 4 \mathrm{H}, \mathrm{N}\left(\mathrm{CH}_{2}\right)_{2}, J=4.6 \mathrm{~Hz}\right), 3.88-3.90\left(\mathrm{t}, 4 \mathrm{H}, \mathrm{O}_{\left(\mathrm{CH}_{2}\right.}\right)_{2}$, $J=4.7 \mathrm{~Hz}), 5.29\left(\mathrm{~s}, 2 \mathrm{H}, \mathrm{NH}_{2}\right), 7.16-7.74(\mathrm{~m}, 9 \mathrm{H}, \mathrm{H}$ ), the signal for $\mathrm{H}-5$ proton may be merged with the aromatic protons; ${ }^{13} \mathrm{C}$ NMR $(\delta \mathrm{ppm}): 47.7 \mathrm{~N}\left(\mathrm{CH}_{2}\right)$, $66.6 \mathrm{O}_{\left(\mathrm{CH}_{2}\right)}, 104.4 \mathrm{C}-5,163.6 \mathrm{C}-2,164.3 \mathrm{C}-6,164.8 \mathrm{C}-4,126.2-138.8-\mathrm{C}_{\text {arom }}$, $146.5,152.3$, ipso-C

4-(3-fluorophenyl)-6-(4-morpholinophenyl)pyrimidin-2-amine (28) IR $(\mathrm{KBr})\left(\mathrm{cm}^{-1}\right): 3437,3204,2965,2928,1623,1597,1225,921,811,649 ;{ }^{1} \mathrm{H}$ NMR ( $\delta$ ppm): $3.33-3.35\left(\mathrm{t}, 4 \mathrm{H}, \mathrm{N}\left(\mathrm{CH}_{2}\right), J=4.7 \mathrm{~Hz}\right), 3.87-3.88\left(\mathrm{t}, 4 \mathrm{H}, \mathrm{O}\left(\mathrm{CH}_{2}\right)\right.$, $J=4.8 \mathrm{~Hz}), 5.27\left(\mathrm{~s}, 2 \mathrm{H}, \mathrm{NH}_{2}\right), 7.28-8.05\left(\mathrm{~m}, 9 \mathrm{H}, \mathrm{H}_{\text {arom }}\right)$, the signal for $\mathrm{H}-5$ proton may be merged with the aromatic protons; ${ }^{13} \mathrm{C}$ NMR $(\delta \mathrm{ppm}): 46.8 \mathrm{~N}\left(\mathrm{CH}_{2}\right)$, $67.4 \mathrm{O}\left(\mathrm{CH}_{2}\right), 103.7 \mathrm{C}-5,163.9 \mathrm{C}-2,164.4 \mathrm{C}-6,164.5 \mathrm{C}-4,125.9-140.3-\mathrm{C}_{\text {arom }}$, 146.6, 154.2 ipso-C.

\section{RESULTS AND DISCUSSION}

(E)-1-(4-morpholinophenyl)-3-aryl-prop-2-en-1-ones (11-19) are synthesized by the condensation of commercially available 1-(4-morpholinophenyl) ethanone and substituted benzaldehyde in the presence of sodium hydroxide in ethanol at $20^{\circ} \mathrm{C}$ for $1 \mathrm{~h}$. Treatment of compounds (11-19) with guanidine nitrate in the presence of lithium hydroxide in refluxing ethanol for $4 \mathrm{~h}$ yields the respective 4-(4-morpholinophenyl)-6-aryl-pyrimidin-2-amines (20-28). The schematic representation and the analytical data of compounds (20-28) are given in Scheme-1 and Table-1, respectively. The importance of the title compounds is due to their potential broad-spectrum microbial activity [17]. The structure of the newly synthesized compounds (20-28) is confirmed by melting point, elemental analysis, MS, FT-IR, one-dimensional NMR $\left({ }^{1} \mathrm{H} \&\right.$ $\left.{ }^{13} \mathrm{C}\right)$ spectroscopic data.

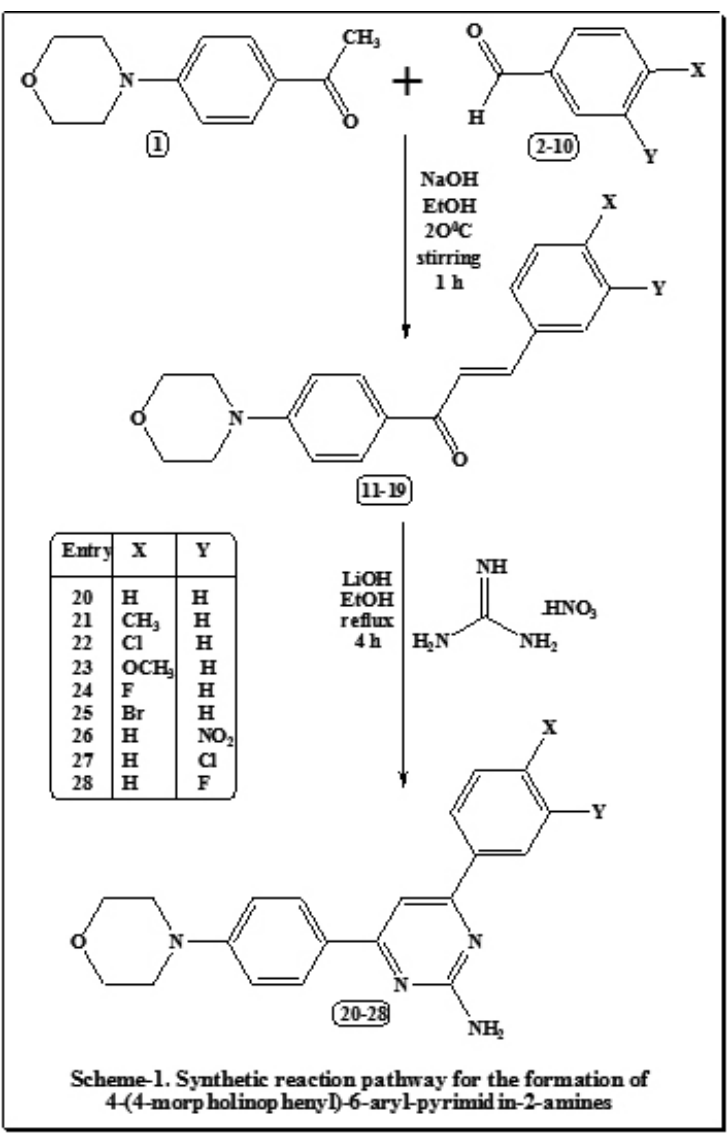



(20-28)

Table 1. Physical and analytical data of (E)-1-4-morpholinophenyl)-3-aryl-prop-2-en-1-ones (11-19) and 4-(4-morpholinophenyl)-6-arylpyrimidin-2-amines

\begin{tabular}{|c|c|c|c|c|c|c|c|c|}
\hline \multirow[b]{2}{*}{ Entry } & \multirow[b]{2}{*}{$\mathbf{X}$} & \multirow[b]{2}{*}{$\mathbf{Y}$} & \multirow[b]{2}{*}{ Yield (\%) } & \multirow[b]{2}{*}{$\mathbf{m} \cdot \mathbf{p}^{\circ} \mathrm{C}$} & \multicolumn{3}{|c|}{ Elemental analysis (\%) } & \multirow{2}{*}{$\begin{array}{c}\mathbf{m} / \mathbf{z} \\
(\mathbf{M}+\mathbf{1})^{+}\end{array}$} \\
\hline & & & & & $\begin{array}{c}\text { C } \\
\text { Found } \\
\text { (calculated) }\end{array}$ & $\begin{array}{c}\text { H } \\
\text { Found } \\
\text { (calculated) }\end{array}$ & $\begin{array}{c}\mathrm{N} \\
\text { Found } \\
\text { (calculated) }\end{array}$ & \\
\hline 11 & $\mathrm{H}$ & $\mathrm{H}$ & 95 & 149 & $\begin{array}{l}77.78 \\
(77.81)\end{array}$ & $\begin{array}{c}6.46 \\
(6.48)\end{array}$ & $\begin{array}{c}4.76 \\
(4.77)\end{array}$ & $\begin{array}{c}294 \\
\mathrm{C}_{19} \mathrm{H}_{19} \mathrm{NO}_{2}\end{array}$ \\
\hline 12 & $\mathrm{CH}_{3}$ & $\mathrm{H}$ & 92 & 179 & $\begin{array}{c}78.15 \\
(78.17)\end{array}$ & $\begin{array}{l}6.81 \\
(6.84)\end{array}$ & $\begin{array}{c}4.54 \\
(4.56)\end{array}$ & $\begin{array}{c}308 \\
\mathrm{C}_{20} \mathrm{H}_{21} \mathrm{NO}_{2}\end{array}$ \\
\hline 13 & $\mathrm{Cl}$ & $\mathrm{H}$ & 90 & 143 & $\begin{array}{c}69.70 \\
(69.72)\end{array}$ & $\begin{array}{c}5.47 \\
(5.50)\end{array}$ & $\begin{array}{c}4.26 \\
(4.28)\end{array}$ & $328 \mathrm{C}_{19} \mathrm{H}_{18} \mathrm{NO}_{2} \mathrm{C}$ \\
\hline 14 & $\mathrm{OCH}_{3}$ & $\mathrm{H}$ & 90 & 111 & $\begin{array}{c}74.29 \\
(74.30)\end{array}$ & $\begin{array}{c}6.48 \\
(6.50)\end{array}$ & $\begin{array}{c}4.31 \\
(4.33)\end{array}$ & $\begin{array}{c}324 \\
\mathrm{C}_{20} \mathrm{H}_{21} \mathrm{NO}_{3}\end{array}$ \\
\hline 15 & $\mathrm{~F}$ & $\mathrm{H}$ & 95 & 160 & $\begin{array}{c}73.29 \\
(73.31)\end{array}$ & $\begin{array}{c}5.76 \\
(5.78)\end{array}$ & $\begin{array}{l}4.48 \\
(4.50)\end{array}$ & $\begin{array}{c}312 \\
\mathrm{C}_{19} \mathrm{H}_{18} \mathrm{NO}_{2} \mathrm{~F}\end{array}$ \\
\hline 16 & $\mathrm{Br}$ & $\mathrm{H}$ & 85 & 145 & $\begin{array}{c}61.27 \\
(61.30)\end{array}$ & $\begin{array}{c}4.83 \\
(4.87) \\
\end{array}$ & $\begin{array}{c}3.74 \\
(3.76) \\
\end{array}$ & $\begin{array}{c}372 \\
\mathrm{C}_{19} \mathrm{H}_{18} \mathrm{BrNO}_{2} \\
\end{array}$ \\
\hline 17 & $\mathrm{H}$ & $\mathrm{NO}_{2}$ & 87 & 135 & $\begin{array}{c}67.43 \\
(67.45)\end{array}$ & $\begin{array}{c}5.29 \\
(5.32)\end{array}$ & $\begin{array}{l}8.26 \\
(8.28)\end{array}$ & $339 \mathrm{C}_{19} \mathrm{H}_{18} \mathrm{~N}_{2} \mathrm{O}_{4}$ \\
\hline 18 & $\mathrm{H}$ & $\mathrm{Cl}$ & 90 & 138 & $\begin{array}{c}69.69 \\
(69.72)\end{array}$ & $\begin{array}{c}5.48 \\
(5.50)\end{array}$ & $\begin{array}{c}4.25 \\
(4.28)\end{array}$ & $328 \mathrm{C}_{19} \mathrm{H}_{18} \mathrm{NO}_{2} \mathrm{Cl}$ \\
\hline 19 & $\mathrm{H}$ & $\mathrm{F}$ & 90 & 154 & $\begin{array}{c}73.28 \\
(73.31)\end{array}$ & $\begin{array}{c}5.74 \\
(5.78)\end{array}$ & $\begin{array}{l}4.47 \\
(4.50)\end{array}$ & $\begin{array}{c}312 \\
\mathrm{C}_{19} \mathrm{H}_{18} \mathrm{NO}_{2} \mathrm{~F}\end{array}$ \\
\hline 20 & $\mathrm{H}$ & $\mathrm{H}$ & 80 & 118 & $\begin{array}{c}72.05 \\
(72.09)\end{array}$ & $\begin{array}{c}6.28 \\
(6.30)\end{array}$ & $\begin{array}{l}16.77 \\
(16.80)\end{array}$ & $\begin{array}{c}333 \\
\mathrm{C}_{20} \mathrm{H}_{21} \mathrm{~N}_{4} \mathrm{O}\end{array}$ \\
\hline 21 & $\mathrm{CH}_{3}$ & $\mathrm{H}$ & 85 & 73 & $\begin{array}{c}72.61 \\
(72.64)\end{array}$ & $\begin{array}{c}6.59 \\
(6.62) \\
\end{array}$ & $\begin{array}{l}16.10 \\
(16.12) \\
\end{array}$ & $\begin{array}{c}347 \\
\mathrm{C}_{21} \mathrm{H}_{23} \mathrm{~N}_{4} \mathrm{O}\end{array}$ \\
\hline 22 & $\mathrm{Cl}$ & $\mathrm{H}$ & 78 & 123 & $\begin{array}{c}65.31 \\
(65.33) \\
\end{array}$ & $\begin{array}{c}5.41 \\
(5.44) \\
\end{array}$ & $\begin{array}{l}15.20 \\
(15.23) \\
\end{array}$ & $\begin{array}{c}367 \\
\mathrm{C}_{20} \mathrm{H}_{20} \mathrm{~N}_{4} \mathrm{OCl}\end{array}$ \\
\hline 23 & $\mathrm{OCH}_{3}$ & $\mathrm{H}$ & 80 & 91 & $\begin{array}{c}69.41 \\
(69.44) \\
\end{array}$ & $\begin{array}{c}6.31 \\
(6.33) \\
\end{array}$ & $\begin{array}{c}15.38 \\
(15.41) \\
\end{array}$ & $\begin{array}{c}363 \\
\mathrm{C}_{21} \mathrm{H}_{23} \mathrm{~N}_{4} \mathrm{O}_{2} \\
\end{array}$ \\
\hline 24 & $\mathrm{~F}$ & $\mathrm{H}$ & 90 & 87 & $\begin{array}{c}68.38 \\
(68.40) \\
\end{array}$ & $\begin{array}{c}5.66 \\
(5.69) \\
\end{array}$ & $\begin{array}{c}15.91 \\
(15.94) \\
\end{array}$ & $\begin{array}{c}351 \\
\mathrm{C}_{20} \mathrm{H}_{20} \mathrm{~N}_{4} \mathrm{OF} \\
\end{array}$ \\
\hline 25 & $\mathrm{Br}$ & $\mathrm{H}$ & 90 & 93 & $\begin{array}{c}58.36 \\
(58.40)\end{array}$ & $\begin{array}{c}4.64 \\
(4.66) \\
\end{array}$ & $\begin{array}{c}13.59 \\
(13.62)\end{array}$ & $\begin{array}{c}411 \\
\mathrm{C}_{20} \mathrm{H}_{19} \mathrm{BrN}_{4} \mathrm{O}\end{array}$ \\
\hline 26 & $\mathrm{H}$ & $\mathrm{NO}_{2}$ & 85 & 176 & $\begin{array}{c}65.92 \\
(65.96)\end{array}$ & $\begin{array}{c}5.47 \\
(5.49) \\
\end{array}$ & $\begin{array}{c}15.35 \\
(15.38)\end{array}$ & $\begin{array}{c}364 \\
\mathrm{C}_{20} \mathrm{H}_{20} \mathrm{~N}_{5} \mathrm{O}_{3}\end{array}$ \\
\hline 27 & $\mathrm{H}$ & $\mathrm{Cl}$ & 80 & 135 & $\begin{array}{c}65.30 \\
(65.33)\end{array}$ & $\begin{array}{c}5.42 \\
(5.44)\end{array}$ & $\begin{array}{l}15.20 \\
(15.23)\end{array}$ & $\begin{array}{c}367 \\
\mathrm{C}_{20} \mathrm{H}_{20} \mathrm{~N}_{4} \mathrm{OCl}\end{array}$ \\
\hline 28 & $\mathrm{H}$ & $\mathrm{F}$ & 78 & 96 & $\begin{array}{c}68.37 \\
(68.40)\end{array}$ & $\begin{array}{c}5.68 \\
(5.69)\end{array}$ & $\begin{array}{l}15.92 \\
(15.94)\end{array}$ & $\begin{array}{c}351 \\
\mathrm{C}_{20} \mathrm{H}_{20} \mathrm{~N}_{4} \mathrm{OF}\end{array}$ \\
\hline
\end{tabular}

\section{ACKNOWLEDGEMENT}

Authors are thankful to NMR Research Centre, Indian Institute of Science, Bangalore for recording spectra. One of the authors namely V.Kanagarajan is grateful to Council of Scientific and Industrial Research (CSIR), New Delhi, Republic of India for providing financial support in the form of CSIR-Senior Research Fellowship (SRF) in Organic Chemistry. J.Thanusu wishes to thank Annamalai University authorities for providing financial support in the form of Research Fellowship.

\section{REFERENCES}

[1] P.D. Jacobus, V. Van Veldhoven, C.W. Lisa, J. Chang, Künzel, Thea Mulder-Krieger, Regina Struensee-Link, M.W. Beukers, I.P. Johannes Brussee, Ijzerman, Bioorg. Med. Chem. 16, 2741 (2008).

[2] T.V. Hughes, S.L. Emanuel, A.K. Beck, S.K. Wetter, P.J. Connolly, Prabha Karnachi, Michael Reuman, Jabed Seraj, A.R. Fuentes-Pesquera, R.H. Gruninger, S.A. Middleton, Ronghui Lin, J.M. Davis, D.F.C. Moffat,
Bioorg. Med. Chem. 17, 3266 (2007).

[3] M.T. Chhabri, H.G. Bhatt, H.G. Raval, P.M. Oza, Bioorg. Med. Chem. Lett. 17, 1022 (2007).

[4] K.L. Sayle, J.F. Bentley, Thomas Boyle, A.H. Calvert, Yuzhu Cheng, N.J. Curtin, J.A. Endicott, B.T. Golding, I.R. Hardcastle, Philip Jewsbury, Veronique Mesguiche, D.R. Newell, M.E.M. Noble, R.J. Parsons, D.J. Pratt, L.Z. Wang, R.J. Griffin, Bioorg. Med. Chem. Lett. 13, 3079 (2003).

[5] Alfredo Pastor, Ramón Alajarin, Vaquero JJ, Julio Alvarez-Builla, Miguel Fau de Casa-Juana, Carlos Sunkel, J.G. Priego, Isabel Fonseca, Julia SanzAparicio, Tetrahedron, 50, 8085 (1994).

[6] M.S. Youssouf, P. Kaiser, G.D. Singh, S. Singh, S. Bani, V.K. Gupta, N.K. Satti, K.A. Suri, R.K. Johri, International Immunopharmacol. 8, 1049 (2008).

[7] Cécile Gasse, Dominique Douguet, Valérie Huteau, Gilles Marchal, Hélène Munier-Lehmann, Sylvie Pochet, Bioorg. Med. Chem. 16, 6075 (2008).

[8] P. Panneerselvam, R.R. Nair, G. Vijayalakshmi, E.H. Subramanian, S.K. Sridhar, Eur. J. Med. Chem. 40, 225 (2005). 
[9] R. Manfred, M. Michael, S. Robert, G. Wolfgang, Eur. Pat. Appl. EP 334146:28. Chem. Abstr. 112, 178999 (1989).

[10] J.J. Hale, S.G. Mills, M. MacCross, C.P. Dorn, P.E. Finke, R.J. Budhu, R.A. Reamer, W.P. Huskey, D. Luffer-Atlas, B.J. Dean, E.M. McGowan, W.P. Feeney, S.H.L. Chiu, M.A. Cascieri, G.G. Chicchi, M.M. Kurtz, S. Sadowski, E. Ber, F.D. Tattersall, N.M.J. Rupniak, A.R. Williams, W. Raycroft, R. Hargreaves, J.M. Metzger, D.E. Maclntyre, J. Med. Chem. 43, 1234 (2000).

[11]M.H. Fisher, M.J. Wyvratt, U.S. Patent 5077290:10. Chem. Abstr. 116, 214513 (1991).

[12]P. Avramova, N. Danchev, R. Buyukliev, T. Bogoslovova, Archiv. der. Pharmazie. 331, 342 (1998).

[13]C.P. Dorn, J.J. Hale, M. MacCoss, S.G. Mills, US Patent 5691336:82, Chem. Abstr. 128, 48231 (1997).
[14] M. Gopalakrishnan, P. Sureshkumar, V. Kanagarajan, J. Thanusu, J. Sulf. Chem. 28, 383 (2007)

[15]M. Gopalakrishnan, J. Thanusu, V. Kanagarajan, Chem. Heterocycl. Comp. 44, 950 (2008).

[16]M. Gopalakrishnan, P. Sureshkumar, J. Thanusu, V. Kanagarajan, M.R. Ezhilarasi, Lett. Org. Chem. 5, 142 (2008).

[17] Thanusu J, Kanagarajan V, Gopalakrishnan M. J. Enz. Inhib. Med. Chem. 25, 347 (2010).

[18] M. Gopalakrishnan, J. Thanusu, V. Kanagarajan, Med. Chem. Res. 2007; 16, 392 (2007).

[19] M. Gopalakrishnan, J. Thanusu, V. Kanagarajan, J. Enz. Inhib. Med. Chem. 22, 709 (2007). 